\title{
Dissecting the EUV spectrum of Capella
}

\author{
By NANCY S. BRICKHOUS E \\ ${ }^{3}$ Harvard-Smithsonian Center for Astrophysics, 60 Garden Street, Cambridge, MA 02138
}

Extreme ultraviolet spectra of Capella, obtained at various orbital phases over the past two years by the $E U V E$ satellite, show strong emission lines from a continuous distribntion of temperatures $\left(\sim 10^{5}-10^{7.3} \mathrm{~K}\right)$. In addition to the strong He II $\lambda 303.8$, the spectra are dominated by emission lines of highly ionized iron. Strong lines of Fe IX, XV, XVI, and XVIII-XXIV are used to construct emission measure distribntions for the individual pointings, which show several striking features, including a minimum near $10^{6} \mathrm{~K}$ and a local maximum at $10^{6.8} \mathrm{~K}$. Furthermore, intensities of the highest temperature lines $\left(T_{e}>10^{7} \mathrm{~K}\right)$ show variations (factors of 2-3) at different orbital phases, while the lower temperature Fe lines show variations of about $30 \%$ or less. The low variability of most of the strong low temperature features motivates a detailed analysis of the summed spectrum. With $\sim 280 \mathrm{ksec}$ of total exposure time, we have measured over 200 emission features with $S / N \geq 3.0$ in the summed spectrum. We report here initial results from the analysis of this spectrum. We can now identify lines of Fe VIII and $\mathrm{X}-\mathrm{XIV}$, as well as a number of electron density and abundance diagnostic lines.

We also report here the first direct measurement of the continuum flux around $\sim 100 \AA$ in a cool star atmosphere with EUVE. The continuum flux can be predicted from the emission measure model based on $\mathrm{Fe}$ line emission, and demonstrates that the $\mathrm{Fe} / \mathrm{H}$ abundance ratio is close to the solar photospheric value.

\section{Introduction}

Capella (Alpha Aurigae; HD 34029) is a bright nearby multiple star system that has been the target of numerous observations over the past two decades in the UV and Xray regions. Under the Guest Observer Program of the EUVE satellite, we have now obtained multiple observations of this source. All of the individual pointings confirm the initial results of the calibration data analysis, reported by Dupree et al. (1993): (a) a continuous distribution of temperatures from the transition region to the hot corona; (b) a minimum in the emission measure distribution (EMD) near $10^{6} \mathrm{~K}$; (c) a local maximum in the EMD at $10^{6.8} \mathrm{~K}$; and, (d) a number of transition region lines that are weak relative to their ultraviolet counterparts. It is the goal of this work to explore the predictive capability of the EMD derived from strong Fe lines. Using the summed spectrum from all the EUVE observations, we have now measured over 200 emission features with signal to noise $(\mathrm{S} / \mathrm{N}) \geq 3$ in the three spectrometers. A more detailed analysis can be found in Brickhouse et al. (1995b).

The analysis of strong EUV Fe lines reveals complex structure in the Capella atmosphere not previously discerned by low resolution X-ray spectroscopy. For example, Swank et al. (1981) characterize a number of cool binary coronae as two-temperature distributions using observations taken with the Einstein Solid State Spectrometer. Even the moderate resolution EXOSAT Transmission Grating Spectrometer was unable to resolve line blends, and thus the multithermal emission models of Lemen et al. (1989) are simple parameterizations reliant on global spectral fitting. Among the early results from EUVE spectroscopy are clean, strong emission lines of Fe IX, XV, XVI, and XVIII-XXIV in a number of bright active binaries (Dupree et al. 1993; Landini \& Monsignori Fossi 1993; Stern et al. 1995). For example, Dupree et al. (1993) report about twenty Fe lines from which an EMD is constructed. While the Capella distribution is indeed reminiscent 
of two dominant temperatures (unlike certain other sources), a continuous distribution is required to fit the EUVE lines.

Given the sharpness of features in Capella's atmosphere and the rich diversity of structures found in other sources (Dupree et al. 1995), a number of questions arise as to the physical conditions at various temperatures in the transition region and corona. Dupree et al. (1993) find evidence for high densities $\left(10^{12}\right.$ to $\left.10^{13} \mathrm{~cm}^{-3}\right)$ from $N_{e}$-sensitive lines of Fe XXI. Brickhouse et al. (1995a) discuss apparent inconsistencies in the densities derived from different line ratios, suggesting that some combination of weak lines, blends, and different source regions for Fe XXI might be responsible. Since these densities at $T_{e} \sim 10^{7} \mathrm{~K}$ imply small emitting volumes and confining magnetic fields of several hundred gauss, the confirmation of such high densities is a strong motivation for longer observations.

At lower temperatures, the EMD has been much less well constrained, relying solely on Fe.IX below $T_{e} \sim 2 \times 10^{6} \mathrm{~K}$ to connect the transition region to the corona. Since the emission measure minimum is at a significantly higher temperature $\left(\sim 10^{6} \mathrm{~K}\right)$ than that of the sun $\left(\sim 10^{5} \mathrm{~K}\right)$, the role of various components in the energy balance of Capella must be quite different from the sun. The summed spectrum now fills in much of the gap in coverage of Fe ionization stages, adding Fe VIII and X-XIV. Many of these lines are also $N_{e}$-sensitive.

Drake et al. (1995) discuss the importance of elemental abundance measurements in stellar coronae for understanding coronal heating, and as Cook et al. (1989) point out, significant variations from solar photospheric abundances can also change the shape of the radiative loss function. The summed spectrum of Capella includes lines from many of the abundant elements; we report here the analysis of emission formed at high temperature from elements other than Fe. Discussion of EUV lines formed in the transition region and near the emission measure minimum is forthcoming (Brickhouse et al. 1995b).

\section{Observations and data reduction}

The individual spectra were acquired over five separate pointings. The extraction techniques, described elsewhere (Dupree et al. 1995a), have been applied to the individual observations separately. The spectra are co-added to produce the summed spectra for a total of $2.82,2.77$, and $2.83 \mathrm{ksec}$ for the SW, MW, and LW spectrometers, respectively. Figure 1 shows these spectra, with the $\mathrm{S} / \mathrm{N}$ curve overplotted. The $\mathrm{S} / \mathrm{N}$ of individual lines is calculated over the entire line profile, and thus is somewhat better than the peak value shown. Since the individual spectra were accumulated under different conditions, the simple summation leads to a minor degradation of the effective spectral resolution. The strong, isolated spectral lines maintain Gaussian profiles, with fits that are generally improved more by higher $\mathrm{S} / \mathrm{N}$ than they are degraded by decreased spectral resolution; they are consistent with the performance characteristics described by Boyd et al. (1994). For individual lines, the centroid and flux determinations, the continuum flux subtraction and the correction for interstellar absorption are done as in Dupree et al. (1993). For blended lines, we require that the line widths, deviations from laboratory wavelengths, and wavelength separations of the individual lines be consistent with the spectrometer characteristics before accepting line identification and fluxes.

Variations during the individual pointings are at the $20 \%$ level. Dupree et al. (1995b) find that the intensities of the highest temperature lines $\left(T_{e}>10^{7} \mathrm{~K}\right)$ show modest variations (factors of 2 to 3 ) with different pointings, while the lower temperature lines $\left(T_{e}<10^{7} \mathrm{~K}\right)$ show variations of about $30 \%$ or less. Ayres (1988) shows that the UV line fluxes are remarkably constant with time as well. While variability in the high 

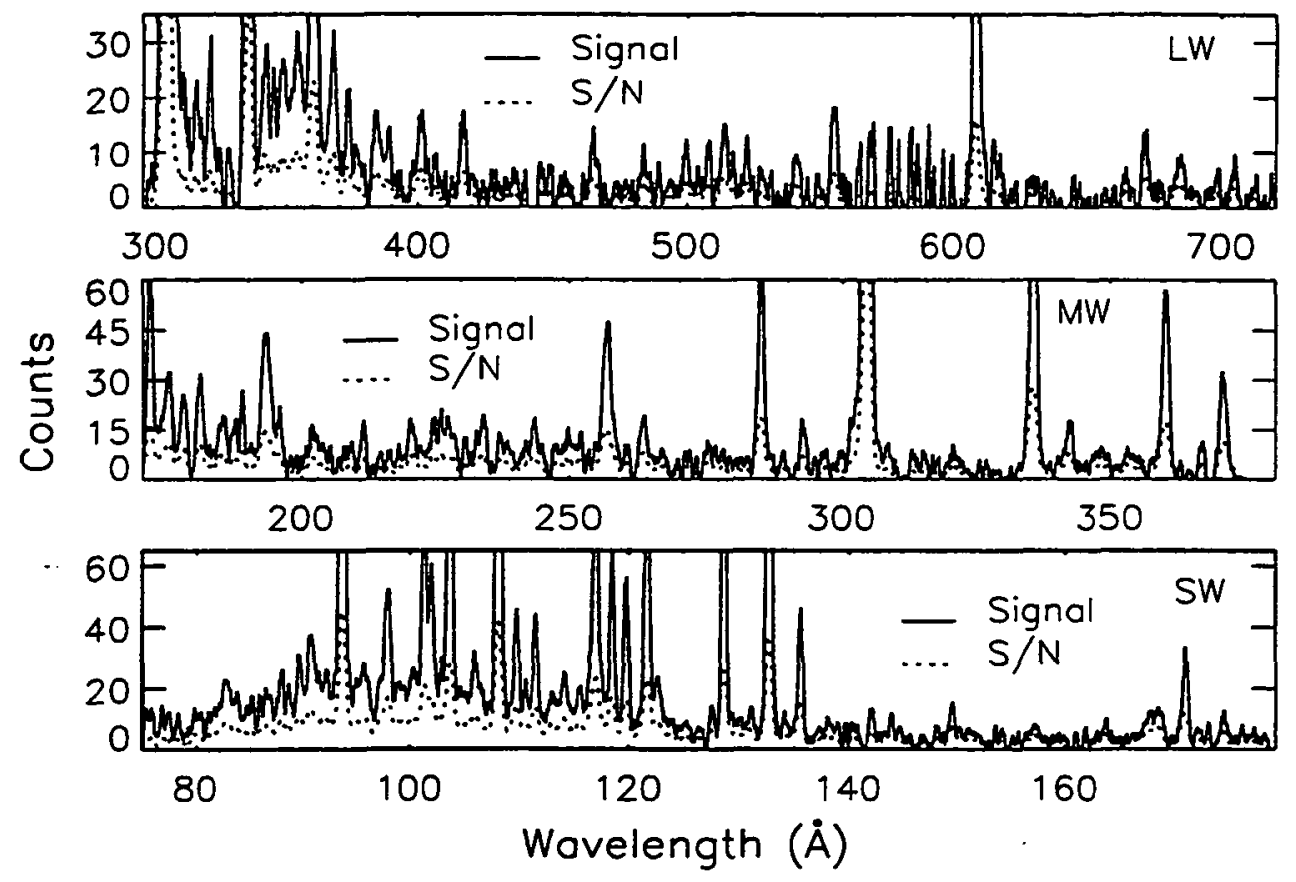

FIgure 1. Capella summed spectrum in the three EUVE spectrometers (solid line); signal to noise (dotted line). Curves are smoothed by 5 and 7 pixels, respectively.

temperature lines is useful for line identification and deblending (such as in the blend of Fe XXIV, Ca XVII, Fe XII, and O V around 192 $\AA$ ), the lack of variability over most of the temperature range gives confidence that this analysis is appropriate for typical atmospheric conditions.

\section{The EMD for the summed spectrum}

The analysis of a coronal source through its EMD provides key insights into heating, cooling, and confinement. While these issues are central to much of the EUV work on stellar coronae, the more immediate goal here is to use the predictive capabilities of an EMD, determined by strong lines from a single element, to examine the rich spectrum for other diagnostics. This goal sets the criterion for the "best fit" as the agreement of strong lines with the model. Figure 2 shows our model for the Capella spectrum, and the agreement of the lines used in constructing it.

For $T_{e} \geq 5 \times 10^{5} \mathrm{~K}$ the EMD depends on the the EUV lines of highly ionized Fe. Newly identified lines are discussed by Brickhouse et al. (1995b). Although the high temperature extent is not well constrained using EUV lines, this model is now consistent with the $A S C A$ continuum above $2 \mathrm{keV}$ (Singh 1994), as well as with the carefully deblended doublet lines of Fe XXIV. We include the X-ray fluxes observed by Vedder \& Canizares (1983) in Figure 2, although we do not use these lines in the fitting. It is interesting to note that the two lines formed predominantly at $T_{e}=10^{6.8} \mathrm{~K}$ (O VIII $\lambda 18.97$ and Fe XVII $\lambda 15.01)$ are in good agreement with the model, while the higher temperature line (Fe XX 212.83 ) exceeds the prediction of the summed spectrum, but is consistent within the range of variability. 

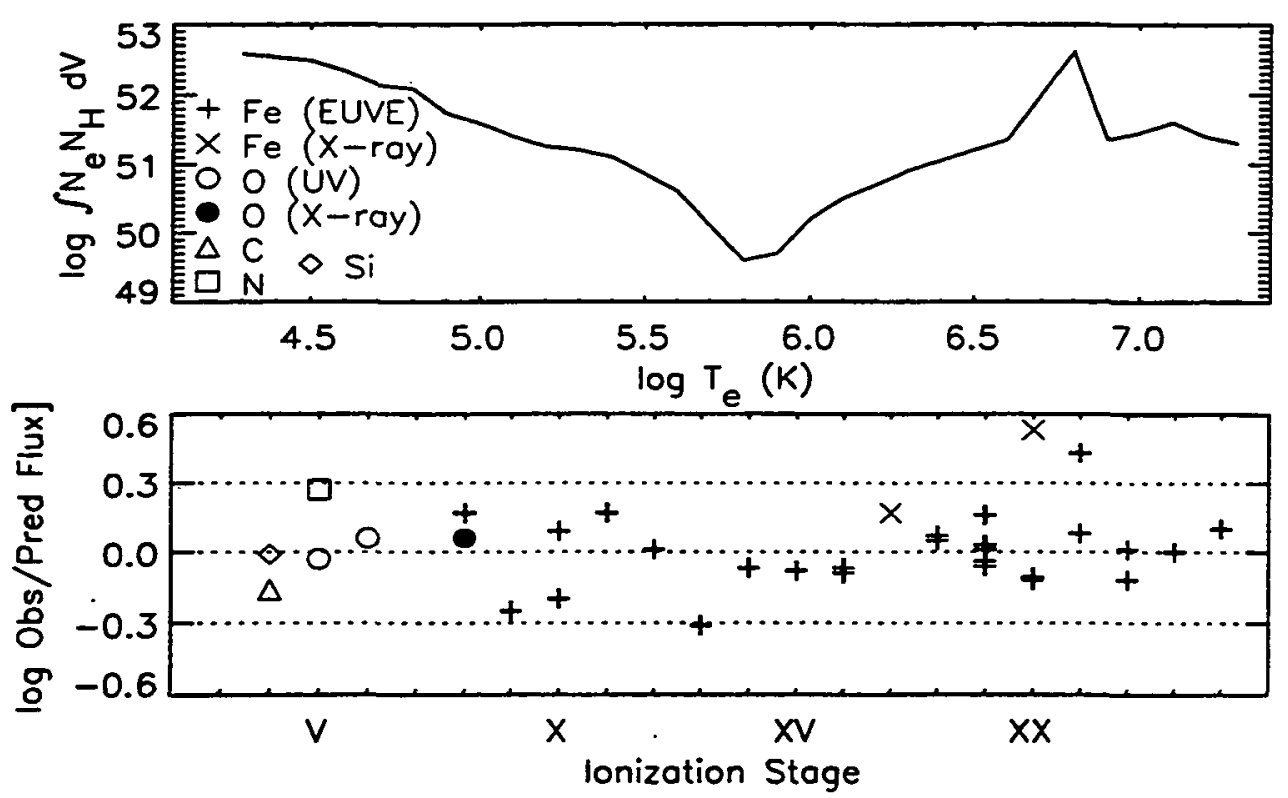

Figure 2. Capella model. Upper: $\log$ Emission Measure $\left(\mathrm{cm}^{-3}\right)$ vs $\log T_{e}(\mathrm{~K})$. Lover: comparison of observed to predicted fluxes for the strong lines. See text for sources of $X$-ray and UV lines from different elements.

Below this temperature we use the total UV line fluxes measured by Linsky et al. (1995) with HST and Hurwitz et al. (1995) with ORPHEUS. As Linsky et al. (1995) discuss, the $\mathrm{N} V$ resonance line flux may reflect $\mathrm{N}$ enrichment in the evolved star, and thus we have forced our fit to the weaker $\mathrm{O} V$ line. Many EUV transition region lines are extremely weak relative to the prediction based on the UV emission lines, as initially reported in Dupree et al. (1993) and discussed in more detail in Brickhouse et al. (1995b).

We cannot emphasize too strongly that the features in the EMD are required by the fluxes of strong lines. For example, Capella's local maximum at $10^{6.8} \mathrm{~K}$ is determined by eight strong lines of Fe XVIII and XIX, for a total of 7600 counts in the summed spectrum. Continuous distribution models which smooth over this feature will underpredict the emission from other lines formed predominantly at this temperature. On the other hand, a two-temperature model has poor predictive powers for lines formed away from the two dominant temperatures. While the single emission measure at $10^{6.8} \mathrm{~K}$ (i.e. the integral over the interval $\Delta T_{e}= \pm 0.05$ dex) in our model accounts for about $80 \%$ of Fe XVIII and XIX line fluxes (with most of the rest of the flux coming from $10^{6.7} \mathrm{~K}$ ), it only accounts for $43 \%, 13 \%$, and $1 \%$ of the Fe XVI, XV, and XIV line fluxes, respectively. Thus many temperatures are required to produce the range of $\mathrm{Fe}$ ionization stages.

With high $\mathrm{S} / \mathrm{N}$ spectra and excellent flux calibration, errors in the atomic models begin to dominate the uncertainties. For the more highly ionized $\mathrm{Fe}$ species, some collision strengths are as accurate as 10\%; for the lower ionization stages (Fe VIII to XIV), collision strengths may be inaccurate at the $30 \%$ level or worse. Dielectronic recombination rates are the largest source of uncertainty in the ionization balance, as discussed by Brickhouse et al. (1995a). In addition to these uncertainties, many of the lower temperature $\mathrm{Fe}$ lines are $N_{e}$-sensitive, and may complicate the emission measure analysis. We find from a synthesis of the Fe XII-XIV spectra that $N_{e} \sim 10^{9} \mathrm{~cm}^{-3}$ is more consistent with observations than $10^{10}$ or $10^{11} \mathrm{~cm}^{-3}$. While we are not able to measure unblended line ratios from these ions, we are able to isolate several line groups. 
At high temperature, line ratio diagnostics give $\log N_{e}\left(\mathrm{~cm}^{-3}\right)=11.1(+0.3$, -no constraint) from Fe XXI $\lambda 128.7 /(\lambda 97.9+\lambda 102.2) ; 12.5(+0.2,-0.1)$ from Fe XXI $\lambda 128.7 /$ $\lambda 113.3 ; 12.35(+0.2,-0.3)$ from Fe XXI $\lambda 128.7 / \lambda 142.2 ; 11.73(+0.2,-0.3)$ from Fe XXI $\lambda 128.7 / \lambda 145.6$; and, 12.05 ( +0.3 , -no constraint) from Fe XXII $\lambda 117.1 / \lambda 114.4$. Uncertainties given correspond to $1-\sigma$ errors in the line ratios. Although the summed spectra provide a great improvement in $\mathrm{S} / \mathrm{N}$ over the calibration data (Dupree et al. 1993), and allow reasonable separation of the blended lines, a large spread remains, which seems to require multiple densities at the source. As noted in Brickhouse et al. (1995a), Fe XXI in the model is formed about equally at $T_{e}=10^{6.8} \mathrm{~K}$ and at $T_{e}>10^{7} \mathrm{~K}$.

\section{Measurement of the continuum and the $\mathrm{Fe} / \mathrm{H}$ abundance ratio}

Stern et al. (1995) measure the continuum emission for Algol by summing the flux in 4 bins to achieve sufficient $S / N$. We are able to measure directly the minimum flux level in the Capella summed spectrum, since $S / N>3.0$ in every bin throughout the wavelength range $81-123 \AA$. Figure 3 is a detail of this spectral region. We attribute the minimum flux to real continuum emission (mostly bremsstrahlung), and compare it to model predictions to derive an $\mathrm{Fe} / \mathrm{H}$ abundance ratio. We find that the $\mathrm{Fe} / \mathrm{H}$ abundance ratio is $0.88 \pm 0.13$ solar, assuming the solar values of Anders \& Grevesse (1989).

The flux in the pixels which contain the lowest flux values determines the continuum, and the quandary is to select the appropriate number of pixels in the flux averaging. We select the lowest points in the spectrum that agree in value with each other to within their statistical error limits. With smoothing by 3 pixels, this condition produces 14 bins to define the continuum, and the derived error is the standard deviation. The value of the continuum defined in this way is consistent with the measurement determined (from fewer points) with smoothing by 5 or 7 pixels. Furthermore, inspection of line lists gives additional confidence that emission lines are avoided. For all but 3 bins, there are no close wavelength matches with lines listed in the Doschek \& Cowan (1984) line list, which includes lines from flaring as well as other solar conditions. We can estimate the contribution from weak, highly ionized $\mathrm{Ni}$ by scaling from lines we observe relative to their theoretical Fe counterparts, for which the tokamak line lists of Dave et al. (1987) and Stratton et al. (1985) are invaluable. The existence of pseudo-continua produced by unknown weak lines is difficult to rule out without models for these weak lines, but their contribution should be well within the errors on the continuum flux measurement.

Figure 3 also shows the temperature-dependence of bremsstrahlung emission. In our model the emission comes predominantly from the $10^{6.8} \mathrm{~K}$ local maximum. The model predicts that the continuum emission decreases by about $20 \%$ over the range from 85 to $140 \AA$; we are not able to determine a slope observationally.

\section{Elemental abundances at high temperature}

The lines used to determine abundances are listed in Table 1. Line emissivities are from Raymond (1988), modified by the atomic data of Zhang et al. (1990) for Li-like ions. Figure 4 shows the dependences of model line emissivities and integrated intensities. For the high $\mathrm{Z}$ (above $\mathrm{Ne}$ ) Li-like lines, the emission in our model comes primarily from $10^{6.8} \mathrm{~K}$. The doublet lines are particularly useful, since their line ratio can give additional confidence in the flux measurement. The $\mathrm{O}$ and $\mathrm{Ni}$ lines in the Table similarly come from this temperature in the model. The errors listed are observational, but there are systematic errors as well, particularly errors in the correction for interstellar absorption. The effect of assuming neutral $\mathrm{He}$ is comparable to the errors in $N_{H}$ given 

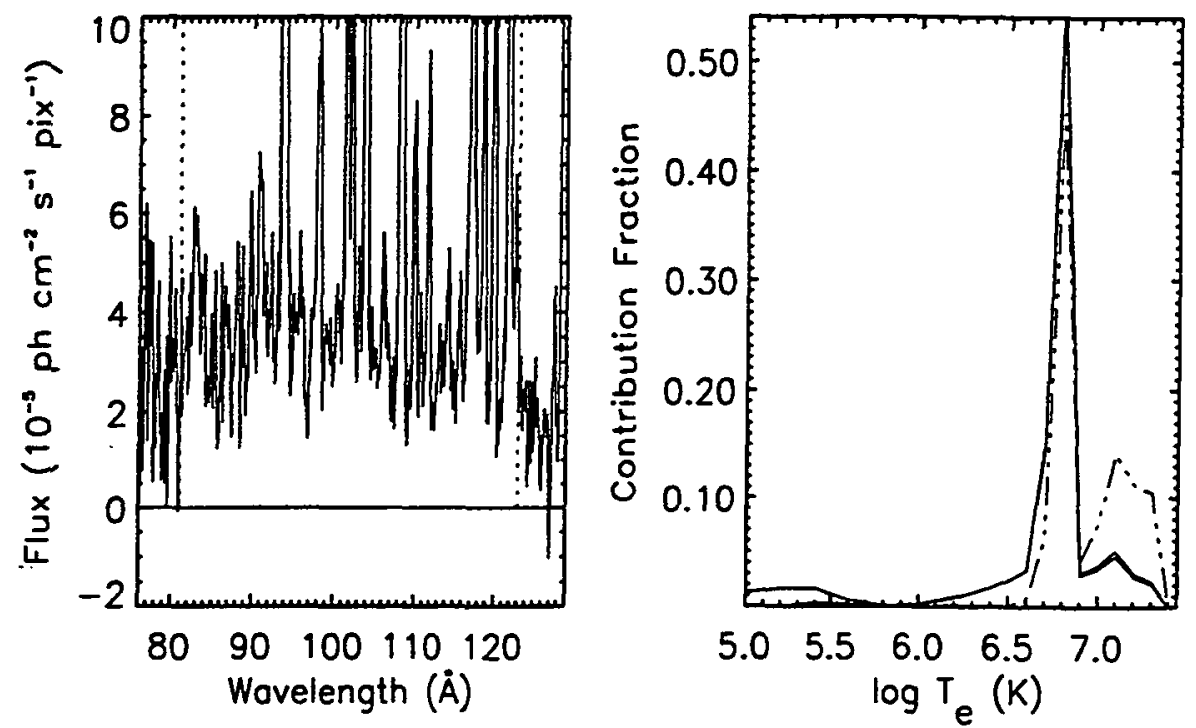

Figure 3. Left: continuum emission in flux units, where I pix $=0.067 \AA$. Dotted vertical lines indicate the wavelength range for which $\mathrm{S} / \mathrm{N}>3$. Right: model of continuum flux at $100 \AA(0.12$ $\mathrm{keV})$ (solid) and at $6 \AA(2.0 \mathrm{keV})$ (dash-dotted) vs temperature. The contribution fraction is the integral of the continuom emissivity over the EMD relative to the total.

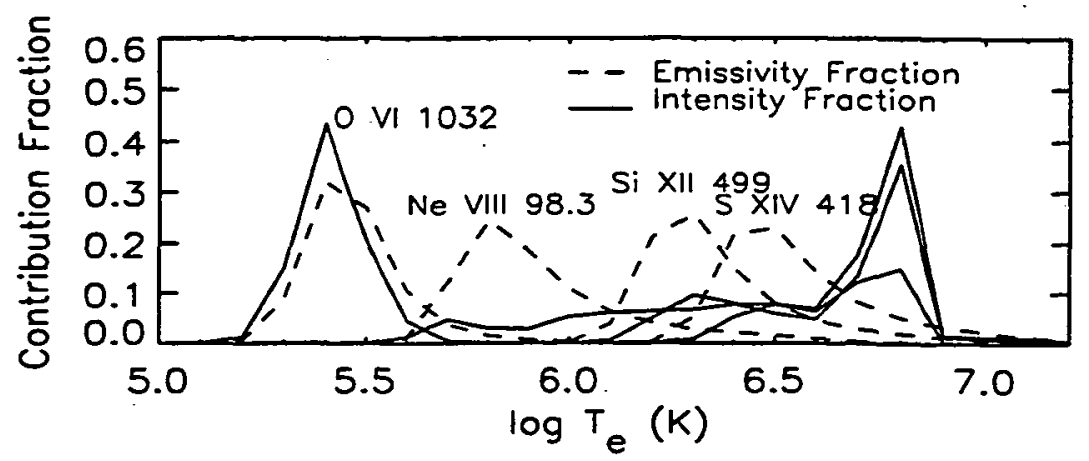

Figure 4. The fractional emissivity (dashed) and intensity (solid contributions as functions of temperature for selected lines from Li-like ions.

by Linsky et al. (1993). Vennes et al. (1993) show that the fraction of ionized He might be as high as $25 \%$. The abundances in Table 1 derived from lines between 227 and $504 \AA$ would then be somewhat reduced. For Si XII the relative abundance would decrease from 1.99 to 1.65 , and would bring the doublet ratio into better agreement with theory.

\section{Conclusions}

High quality EUVE spectra of cool stars, such as the summed spectrum of Capella, are rich with information on the physical conditions in the atmosphere. Long observations confirm the presence in Capella of high densities $\left(\geq 10^{12} \mathrm{~cm}^{-3}\right)$ at high temperature, but lower densities are present as well at other temperatures. We have now measured the relative abundances of a number of elements which emit radiation at the local maximum 


\begin{tabular}{|c|c|c|c|c|c|}
\hline & Element & Emission Lines & Capella ${ }^{\dagger}$ & Capella $\stackrel{+}{+}$ & Solar Corona $\$$ \\
\hline \multirow{2}{*}{\multicolumn{2}{|c|}{0}} & O VIII $\lambda 102.45^{\prime \prime}$ & $0.42 \pm 0.18$ & \multirow[t]{2}{*}{$0.13(0.03,0.27)$} & \multirow[t]{2}{*}{0.9} \\
\hline & & O VIII $\lambda 18.97^{\dagger \dagger}$ & $1.02 \pm 0.28$ & & \\
\hline & $\mathrm{Si}$ & $\begin{array}{l}\text { Si XII } \lambda 499.40 \\
\text { Si XII } \lambda 520.67\end{array}$ & $1.99 \pm 0.38$ & $0.85(0.67,1.07)$ & 3.6 \\
\hline & $S$ & $\begin{array}{l}\text { S XIV } \lambda 417.61 \\
\text { S XIV } \lambda 445.77\end{array}$ & $1.15 \pm 0.18$ & \multirow[t]{2}{*}{$0.73(0.39,1.14)$} & 1.1 \\
\hline & Ar & $\begin{array}{l}\text { Ar XVI } \lambda 353.92 \\
\text { Ar XVI } \lambda 389.14\end{array}$ & $2.41 \pm 0.31$ & & 1.0 \\
\hline & $\mathrm{Fe}$ & (all) & $0.88 \pm 0.13$ & \multirow[t]{2}{*}{$0.46(0.36,0.61)$} & 2.7 \\
\hline & $\mathrm{Ni}$ & $\begin{aligned} \text { Ni XVII } & \lambda 249.18 \\
N i \text { XVIII } & \lambda 291.97 \\
\text { Ni XVIII } & \lambda 320.54\end{aligned}$ & $1.81 \pm 0.20$ & & 3.9 \\
\hline \multicolumn{6}{|c|}{ TaBLE 1. Elemental Abundances at $T_{e} \sim 10^{6.8} \mathrm{~K}^{\ddagger \ddagger}$} \\
\hline \multicolumn{6}{|c|}{$\begin{array}{l}\dagger \text { This work. Errors are } 1-\sigma \text { estimates of measurement uncertainties, including statistical } \\
\text { errors, deblending of weak lines and high order flux subtraction. }\end{array}$} \\
\hline \multicolumn{6}{|c|}{$\begin{array}{l}\mp \text { From Drake et al. (1994). Allowed ranges reflect } 90 \% \text { confidence levels from X-ray spectral } \\
\text { fitting with two temperatures. }\end{array}$} \\
\hline \multicolumn{6}{|c|}{ I Feldman (1992) } \\
\hline \multicolumn{6}{|c|}{$\begin{array}{l}\text { It Observed by Vedder \& Canizares (1983). Prediction uses the EMD from this work. } \\
\text { \# Abundances are relative to solar photospheric abundances of Anders \& Grevesse (1989). }\end{array}$} \\
\hline
\end{tabular}

at $\sim 10^{6.8} \mathrm{~K}$, and find that they are more consistent with solar photospheric than coronal values. Density and abundance measurements may provide keys to understanding the nature of this enhanced emission region. Variability in the $N_{e}$-sensitive lines at the highest temperatures may provide a tool for distinguishing different sources of emission.

The author appreciates the contributions of Andrea Dupree, John Raymond and Greg Hanson. This work has been supported by NAGW-528 and NAG5-2330 to the Smithsonian Institution.

\section{REFERENCES}

ANDERS, E., \& Grevesse, N. 1989 Abundances of the Elements: Meteoritic and Solar. Geochim. Cosmochim. Acta 53, 197-214.

AYres, T. R. 1988 A Spectral Dissection of the Ultraviolet Emissions of Capella. Ap. J. 331, $467-476$.

Boyd, W., Jelnsky, P., Fniey, D. S., Dupuis, J., Abbott, M., Christan, C., \& MAIDNA, R. F. 1994 In-Orbit Performance of the Spectrometers of the Extreme Utraviolet Explorer. Proc. SPIE, 2280, 280-296.

Brickhouse, N. S., Raymond, J. C., \& SMTtr, B. W. 1995a New Model of Iron Spectra in the Extreme Ultraviolet and Application to SERTS and EUVE Observations: A Solar Active Region and Capella. Ap. J. Supp. 97, 551-570.

Brickrouse, N. S., Dupree, A. K., \& RaYmoNd, J. C. 1995b Analysis of the EUVE Spectrum of Capella from the Transition Region to the Hot Corona. In progress. 
Cook, J. W., Cheng, C.-C., Jacobs, V. L., \& Antrochos, S. K. 1989 Effect of Coronal Elemental Abundances on the Radiative Loss Function. Ap. J. 338, 1176-1183.

Dave et al. 1987 Time-resolved spectra in the 80-340 $\AA$ Wavelength Region from Princeton Large Torus Tokamak Plasmas., J. Opt. Soc. Am. B4, 635-643.

Doschex, G. A., \& Cowan, R. D. 1984 A Solar Spectral Line List Between 10 and $200 \AA$ Modified for Application to High Spectral Resolution X-ray Astronomy. Ap. J. Supp. 56 67-89.

Drake, J. J., Lameng, J. M., \& Wming, K. G. 1995 Stellat Coronal Abundances. II. The First Ionization Potential Effect and Its Absence in the Corona of Procyon. Ap. J. 443, $393-415$.

Drake, S. A., Singh, K. P., White, N. E., \& Smon, T. 1994 ASCA X-ray Spectra of the Active Single Stars $\beta$ Ceti and $\Pi^{1}$ Ursae Majoris. Ap. J. 436, L87-L90.

Dupree, A. K., Brickzouse, N. S., Doschek, G. A., Green, J. C., \& Raymond, J. C. 1993 The Extreme Ultraviolet Spectrum of Alpha Aurigae (Capella). Ap. J. 418, L41-L44.

Dupree, A. K., Brickrouse, N. S., \& Hanson, G. J. 1995a High Temperature Structure in Cool Binary Stars. This proceedings.

Dupree, A. K., Brickrouse, N. S., Doschex, G. A., Hanson, G. J., \& Raymond, J. C. 1995b EUVE Spectra of Alpha Aurigae (Capella) at Different Phases. In preparation.

Ferdman, U. 1992 Elemental Abundances in the Upper Solar Atmosphere. Phys. Scripta 46, 202-220.

HurwTtz, M., BowYer, S., \& Dupree, A. K. 1995 ORPHEUS Observations of Capella. In preparation.

Landini, M., \& Monsignori Fossi, B. C. 1993 Extreme Ultra Violet Plasma Diagnostic: a Test Using EUVE Calibration Data. Astron. Astrophys. 275, L17-L20.

Lemen, J. R., Mewe, R., SchrJver, C. J., \& FuUdra, A. 1989 Coronal Activity in F-, G-, and K-Type Stars. III. The Coronal Differential Emission Measure Distribution of Capella, $\sigma^{2} \mathrm{CrB}$, and Procyon. Ap. J. 341, 471-483.

LnssKY, J. L et al. 1993 Goddard High-Resolution Spectrograph Observations of the Local Interstellar Medium and the Deuterium/Hydrogen Ratio along the Line of Sight toward Capella. Ap. J. 402, 694-709.

Linsky, J. L., WoOd, B. E., Judge, P., Brown, A., ANdrulis, C., \& AYres, T. R. 1995 The Transition Regions of Capella. Ap. J., 442, 381-400.

RAYmond, J. C. 1988 Radiation from Hot, Thin Plasmas. in R. Pallavicini, ed, Hot Thin Plasmas in Astrophysics (Dordrecht: Kluwer), 3-20.

SINGE, KP 1994, private communication.

Stern, R. A., Lemen, J. R., SchmTt, J. H. M. M., \& PYe, J. P. 1995 EUVE Observations of Algol: Detection of a Continuum and Implications for the Coronal [Fe/H] Abundance. Ap. J., 444, L45-L48.

S Trutton et al. 1985 Relative Intensities of $2 \mathrm{~s}^{2} 2 \mathrm{p}^{k}-2 \mathrm{~s} 2 \mathrm{p}^{k+1}$ transitions in FI- to BI-like Ti, $\mathrm{Cr}, \mathrm{Fe}, \mathrm{Ni}$, and $\mathrm{Ge}$ in a Tokamak Plasma: A Comparison of Experiment and Theory., Phys. Rev. A 31, 2534-2547.

Swank, J. H., White, N. E., Holt, S. S., \& Becker, R. H. 1981 Two-Component X-ray Emission from RS Canum Venaticorum Binaries. Ap. J. 246, 208-214.

Vedder, P. W., \& Canizargs, C. R. 1983 Measurement of Coronal X-ray Emission Lines from Capella. Ap. J. 270, 666-670.

Vennes, S. et al. 1993 The First Detection of Ionized Helium in the Local ISM: EUVE and IUE Spectroscopy of the Hot DA White Dwarf GD 246. Ap. J. 410, L119-I122.

Zhang, H. I., SAmpson, D. H., \& Fontes, C. J. 1990 Relativistic Distorted-Wave Collision Strengths and Oscillator Strengths for the $85 \mathrm{Li}$-like Ions with $8 \leq Z \leq 92$. At. Data Nucl. Data Tables, 44, 31-77. 